\title{
Kodaira dimensions and hyperbolicity of nonpositively curved compact Kähler manifolds
}

Fangyang Zheng

\begin{abstract}
In this article, we prove that a compact Kähler manifold $M^{n}$ with real analytic metric and with nonpositive sectional curvature must have its Kodaira dimension, its Ricci rank and the codimension of its Euclidean de Rham factor all equal to each other. In particular, $M^{n}$ is of general type if and only if it is without flat de Rham factor. By using a result of Lu and Yau, we also prove that for a compact Kähler surface $M^{2}$ with nonpositive sectional curvature, if $M^{2}$ is of general type, then it is Kobayashi hyperbolic.
\end{abstract}

Mathematics Subject Classification (2000). Primary 53C55, Secondary 53C12.

Keywords. Compact Kähler manifold, nonpositive sectional curvature, Ricci rank, Ricci kernel foliation, Euclidean de Rham factor, Kobayashi hyperbolic, Kähler hyperbolic, visibility axiom.

\section{Introduction}

Nonpositively curved Riemannian manifolds have been one of the focal points in differential geometry since the 1980's. [B-G-S], [E-H-S], [B], [Bu-S], [E3], [E4] and the references therein provide a good view on the subject. The complex special case, namely, Kähler manifolds with nonpositive sectional curvature, have received far less attention, with only a few exceptions ([Si], [M], [M1], [M2], [MSY], [G], etc.). [G] gives a good discussion on the Kähler case.

For convenience, let us denote by $\mathcal{F}_{n}$ the set of all compact Kähler manifolds of complex dimension $n$ with nonpositive sectional curvature.

On one hand, $\mathcal{F}_{n}$ is a much more restrictive class than its parent set of all nonpositively curved compact Riemannian manifolds. Such manifolds tend to be very rigid. For instance, Siu [Si] proved the strong rigidity (of the Kählerian complex structure) under a slightly stronger curvature assumption. Because of this restrictiveness, there is a better chance to obtain more precise characterization and stronger structural statements in the Kähler case than in the Riemannian case.

On the other hand, it is a general belief that the set $\mathcal{F}_{n}$ is quite large, and con-

\footnotetext{
Research partially supported by a NSF Grant and an Alfred P. Sloan Fellowship. This project is also sponsored by the National Security Agency under grant \# MDA 904-98-1-0036.
} 
tains rich examples. [Z1] and [Z2] provided some evidence on this point, especially in complex dimension 2 . So we are not dealing with small or nearly empty set of specimen here.

In the theory of nonpositively curved Riemannian manifolds, one of the main issues is to examine the fine distinction between the behavior of negatively curved and nonpositively curved manifolds. In the Kähler case, we propose the following conjectures which are simply based upon intuition:

Conjectures. Let $\left(M^{n}, g\right)$ be in $\mathcal{F}_{n}$.

1. The Kodaira dimension of $M$ is equal to the rank of its Ricci form, and is equal to the codimension of its Euclidean de Rham factor. In particular, $M$ is of general type if and only if it has trivial Euclidean de Rham factor.

2. If $M$ is of general type, then it is Kobayashi hyperbolic.

3. If $M$ is of general type and is irreducible (i.e., no finite cover of $M$ is biholomorphic to a product), and is not a locally Hermitian symmetric space of rank $\geq 2$, then it satisfies the visibility axiom (i.e., the universal cover $\widetilde{M}$ does not contain any 2-flat).

Let us first have a brief discussion about these three conjectures. The first one will be implied by the following (slightly stronger) statement. If $\widetilde{M}$ is a simplyconnected, complete Kähler manifold with nonpositive sectional curvature and with trivial Euclidean de Rham factor, then the Ricci curvature must be negative definite at some point. This is in fact true if the metric is real analytic, as we shall see later. However, its Riemannian analogue is not true. We shall see such examples in Section 4.

For the second part, one can raise a slightly stronger conjecture by asserting that $M$ is Kähler hyperbolic (in the sense of Gromov $[\mathrm{G}]$ ). Note that Kähler hyperbolicity implies Kobayashi hyperbolicity, but is stronger than the latter (for example, Kähler hyperbolicity implies that $\pi_{1}(M)$ is of exponential growth. On the other hand, there are examples of compact complex manifold with $\pi_{1}=0$ that are Kobayashi hyperbolic, e.g., a generic surface with large degree in $\mathbf{C P}^{3}$ ).

By Brody's theorem, a compact complex manifold $M$ will be Kobayashi hyperbolic if any holomorphic map $f: \mathbf{C} \rightarrow M$ is constant. When $M$ is nonpositively curved and of general type, it does not contain any rational or elliptic curve. So Conjecture 2 is essentially about 'closing up' the holomorphic 2-flats in $M$. In the Riemannian case, such questions were studied extensively by Schroeder et al. ([A-S], [B-S], [H-S] and [S]-[S3]).

For the third conjecture, first of all, a general type manifold $M^{n}$ in $\mathcal{F}_{n}$ always has $c_{1}<0$, as we shall see later. Hence $M$ admits a Kähler-Einstein metric by [Y]. According to Frankel [Fr], there exists a finite cover $M^{\prime}$ of $M$ such that

$$
M^{\prime}=M_{0} \times M_{1} \times \cdots \times M_{k}
$$

where $M_{0}$ is a locally Hermitian symmetric space, while for each $1 \leq i \leq k, M_{i}$ is a irreducible (i.e., any finite cover of it is not biholomorphic to the product of 
two complex manifolds of positive dimensions) and its universal cover has discrete automorphism group.

The assumption in Conjecture 3 simply says that, either $M$ is a ball quotient, or it is irreducible with $\operatorname{Aut}(\widetilde{M})$ being discrete. Note that if $\widetilde{M}$ is a product, then the product of a pair of geodesics from different factors gives a 2 -flat.

The conclusion of Conjecture 3 is equivalent to any one of the following:

- $M$ satisfies the visibility axiom, or

- Any two distinct points in the boundary $\widetilde{M}(\infty)$ is the two ends of a geodesic in $\widetilde{M}$ (i.e., the Tits metric on $\widetilde{M}(\infty)$ is degenerate: $T d(x, y)=+\infty$ whenever $x \neq y$ ), or

- $\pi_{1}(M)$ is a hyperbolic group ([G1]), or

- $M$ is real hyperbolic in the sense of Gromov, i.e., any absolutely minimizing conformal map from $\mathbf{R}^{2}$ into $M$ is constant. Here 'absolutely minimizing' means that no homotopic map fixed outside a compact set of $\mathbf{R}^{2}$ can decrease the area.

The main purpose of this article is to confirm Conjecture 1 under the additional assumption that the metric $g$ is real analytic. We also give a proof of Conjecture 2 in the case of complex dimension 2, by making use of a result due to $\mathrm{Lu}$ and Yau $([\mathrm{L}-\mathrm{Y}])$. We hope our discussion here can generate some interests towards these conjectures and some related questions. To close this introduction part, we state the following:

Theorem 1. If $(M, g)$ is in $\mathcal{F}_{n}$ and $g$ is real analytic, then the Kodaira dimension of $M$ is equal to the (complex) codimension of the Euclidean de Rham factor of $M$, and is equal to the maximum rank of its Ricci $(1,1)$-form. In particular, $M$ is of general type if and only if it has trivial Euclidean de Rham factor.

Theorem 2. If $\left(M^{2}, g\right)$ is in $\mathcal{F}_{2}$ and $M$ is of general type, then it is Kobayashi hyperbolic.

\section{Preliminaries}

In this section, we collect some facts which will be needed in our proofs.

\subsection{Euclidean de Rham factors}

First let us recall the work of Eberlein on Euclidean de Rham factors ([E1], [E2]). Suppose $M$ is a compact Riemannian manifold with nonpositive sectional curvature. Then $M$ has non-trivial Euclidean de Rham factor if and only if $\pi_{1}(M)$ has non-trivial center. In this case there is a finite cover $M^{\prime}$ of $M$ and a Riemannian fiber bundle $f: M^{\prime} \rightarrow N$ with flat torus fiber onto a compact Riemannian manifold $N$. $N$ also has nonpositive sectional curvature but has trivial Euclidean de Rham 
factor. The fibers of $f$ correspond to the Euclidean de Rham factor of $M . M^{\prime}$ is diffeomorphic (but may not be isometric) to the product of the fiber with $N$.

When $(M, g)$ is in $\mathcal{F}_{n}, f$ is a holomorphic fiber bundle with typical fiber being a flat complex torus, and the base $N^{k}$ is in $\mathcal{F}_{k}$ with trivial Euclidean de Rham factor. Here $k$ is the (complex) dimension of $N$, which is of course just the codimension of the Euclidean de Rham factor of $M$.

\subsection{Totally geodesic submanifolds and their parallels}

In this subsection let us recall some well known facts about complete totally geodesic submanifolds and their parallels in a Cartan-Hadamard manifold. See [B-G-S] or $[\mathrm{B}-\mathrm{S}]$ for more details.

Let $\widetilde{M}$ be a complete, simply-connected Riemannian manifold with nonpositive sectional curvature. For a subset $A \subseteq \widetilde{M}$, let $T u b e_{a}(A)=\{x \in \widetilde{M} \mid d(x, A) \leq a\}$ be the $a$-tube of $A$. Two complete totally geodesic submanifolds $Y$ and $Y^{\prime}$ of $\widetilde{M}$ are called parallel, if the Hausdorff distance between $Y$ and $Y^{\prime}$ is finite, i.e., if there exists $a$ such that $Y \subseteq T u b e_{a}\left(Y^{\prime}\right)$ and $Y^{\prime} \subseteq T u b e_{a}(Y)$. By the Sandwich Lemma, two parallel complete totally geodesic submanifolds $Y, Y^{\prime}$ bound a convex subset isometric to $Y \times[0, a]$, where $a$ is the Hausdorff distance between $Y$ and $Y^{\prime}$.

Now for a complete totally geodesic submanifold $Y$, let $P_{Y}$ be the set of all points in $\widetilde{M}$ which lie on the parallels of $Y$. Then $P_{Y}$ is convex and splits isometrically as $P_{Y}=Y \times Z$, where $Z$ is a convex subset of $\widetilde{M}$. If the metric of $\widetilde{M}$ is real analytic, then $P_{Y}$ is without boundary.

\subsection{The kernel foliation of the Ricci tensor}

We shall need the following lemma, the proof of which follows directly from the structure equations and the second Bianchi identity, and is omitted.

Lemma 1. Let $M$ be a Riemannian manifold with nonpositive sectional curvature. Assume that the Ricci curvature tensor is nowhere negative definite. For $x \in M$, denote by $L_{x} \subseteq T_{x} M$ the kernel of the Ricci tensor. Let $U$ be the open subset where $L_{x}$ has constant minimum dimension and $\mathcal{L}$ the distribution in $U$ defined by $L_{x}$. Then $\mathcal{L}$ is a totally geodesic foliation in $U$ with flat leaves.

We will call this $\mathcal{L}$ the kernel foliation of the Ricci tensor. It is actually equal to the nullity foliation of the Riemannian curvature tensor, that is, at any $x \in U$, $X \in L_{x}$ if and only if $R(X, Y, Z, W)=0$ for any $Y, Z, W$ in $T_{x} M$. So by the result of Ferus [Fe], when $M$ is complete, any leaf of $\mathcal{L}$ will be complete submanifold in $M$.

\subsection{General type manifolds in $\mathcal{F}_{n}$}

Now let $(M, g)$ be a manifold in $\mathcal{F}_{n}$. Denote by $R i c_{g}$ the Ricci $(1,1)$-form of $g$. It is nonpositive everywhere on $M$. Let $K_{M}$ be the canonical line bundle of $M$. 
If $M$ is of general type, then $K_{M}$ must be ample by the result of [K], since $M$ does not contain any rational curves. Hence $K_{M}^{n}=\int_{M}\left(-R i c_{g}\right)^{n}>0$. Therefore $R i c_{g}$ must be negative definite somewhere. On the other hand, if $R i c_{g}$ is negative definite somewhere in $M$, then the generalized Kodaira embedding theorem (cf. [S-S]) implies that $M$ is of general type. In conclusion, one has:

Lemma 2. Let $(M, g)$ be a manifold in $\mathcal{F}_{n}$. Then $M$ is of general type if and only if the Ricci form Ric $c_{g}$ is negative definite somewhere.

Proof of Theorem 1. Let $\left(M^{n}, g\right)$ be in $\mathcal{F}_{n}$ with real analytic $g$. By Eberlein's theorems, there exists a finite cover $M^{\prime}$ of $M$ and a holomorphic fiber bundle $f: M^{\prime} \rightarrow N$ such that the fiber is a flat complex torus, and the base $N$ is in $\mathcal{F}_{r}$ and has trivial Euclidean de Rham factor. The metric of $N$ is also real analytic since the universal cover of $N$ is just the product of all the non-Euclidean de Rham factors of $M$. On one hand, the Kodaira dimension of $M, M^{\prime}$ and $N$ are all equal. On the other hand, $M$ and $N$ have the same 'Ricci rank' and the same 'codimension of the Euclidean de Rham factor'. So Theorem 1 is proved if we can show that $N$ is of general type, i.e., its Kodaira dimension is equal to its dimension.

Assume the contrary, then by Lemma 2 above, the Ricci tensor of $N$ is nowhere negative definite. The following Theorem 3 then says that $N$ will have a nontrivial Euclidean de Rham factor, a contradiction. This will complete the proof of Theorem 1.

Theorem 3. Let $\left(M^{n}, g\right)$ be a complete, simply-connected Kähler manifold with nonpositive sectional curvature and with $g$ being real analytic. If the maximum rank of the Ricci tensor is equal to $r<n$, then $M$ has a non-trivial Euclidean de Rham factor $\mathbf{C}^{n-r}$.

\section{The Proof of Theorem 3}

In this section, we shall prove Theorem 3, thus completing the proof of Theorem 1. Throughout this section, $\left(M^{n}, g\right)$ will be a complete, simply-connected Kähler manifold with real analytic metric $g$ and with nonpositive sectional curvature. The maximum rank of the Ricci tensor is $r$, and we assume that $r<n$. Denote by $U$ the open (dense) subset where the Ricci tensor has rank $r$, and by $\mathcal{L}$ the distribution in $U$ given by the kernel of the Ricci tensor. As noted before, $\mathcal{L}$ is a foliation, whose leaves are flat, complete, totally geodesic complex submanifolds in $M$.

Denote by $\mathcal{L}^{\perp}$ the distribution in $U$ representing the orthogonal complement of $\mathcal{L}$, so we have $T_{M}=\mathcal{L} \oplus \mathcal{L}^{\perp}$ in $U$, where $T_{M}$ stands for the holomorphic tangent bundle.

Let us denote by $T M=\mathcal{F} \oplus \mathcal{F}^{\perp}$ the corresponding decomposition, where $T M$ 
is the real tangent space (of real dimension $2 n$ ), and $\mathcal{F}$ is real underlying foliation of $\mathcal{L}$. It is of real rank $2 r$.

Recall that the conullity operator of a totally geodesic foliation $\mathcal{F}$ in a Riemannian manifold is defined by (cf. [A], [D-R])

$$
C_{T}(X)=-\left(\nabla_{X} \tilde{T}\right)^{\perp}
$$

where $T$ and $X$ are tangent vectors in $\mathcal{F}$ and $\mathcal{F}^{\perp}$, respectively, and $\tilde{T}$ is a local vector field in $\mathcal{F}$ extending $T$. Here $Y^{\perp}$ stands for the $\mathcal{F}^{\perp}$-component of $Y$. It is well defined, and satisfies the Riccati type equation

$$
\nabla_{T} C_{S}=C_{S} \circ C_{T}-C_{\nabla_{T} S}-\{R(T, \cdot) S\}^{\perp}
$$

for any two vector fields $T$ and $S$ in $\mathcal{F}$. In our case, the curvature term vanishes, and if we choose $S$ to be parallel in each leaf of $\mathcal{F}$, then the above equation becomes

$$
\nabla_{T} C_{S}=C_{S} \circ C_{T}
$$

In particular, along any geodesic $\gamma(t)$ contained in a leaf $F$ of $\mathcal{F}$, one has

$$
\nabla_{T} C_{T}=\left(C_{T}\right)^{2}
$$

where $T=\gamma^{\prime}(t)$. Since each $F$ is complete by Ferus' theorem [Fe], we know that $C_{T}$ for any $T \in \mathcal{F}$ cannot have non-zero real eigenvalues (cf. [D-R]). Note that here and below we regard $C_{T}$ as linear transformations on the finite dimensional vector space of vector fields in $\mathcal{F}^{\perp}$ that are parallel along $\gamma(t)$, with $\gamma^{\prime}(t)=T$.

Abe used the conullity operators to establish, among other things, the complex analogue of the classic cylinder theorem of Hartman-Nirenberg, which states that a complete, developable (real or complex) Euclidean submanifold with Gauss rank 1 must be a cylinder. In [D-R], Dajczer and Rodriquez used these operators to show that, if $M^{2 n}$ is a complete Kähler manifold and $f: M^{2 n} \rightarrow \mathbf{R}^{N}$ is a minimal immersion, with Gauss rank $r \leq 4$ (i.e., the image of Gauss map of $f(M)$ has real dimension $r \leq 4)$, then either $M^{2 n}=N^{4} \times \mathbf{R}^{2 n-4}$ and $f$ is a cylinder: $f=f_{1} \times i d$, or $M^{2 n}$ admits a complex foliation whose leaves are isometrically mapped by $f$ onto affine subspaces $\mathbf{R}^{2 n-2}$.

We refer the readers to $[\mathrm{A}]$ and $[\mathrm{D}-\mathrm{R}]$ for a detailed discussion ob these conullity operators. However, we would like to point out that, in the extrinsic case (e.g., Euclidean submanifolds), the fact that $C_{T}$ needs to be symmetric with respect to the second fundamental form makes it more restrictive (e.g., when $M$ is Kähler, all $C_{T}$ are complex linear) than the general (intrinsic) case. The proofs in [D-R] is quite different than here, because things are extrinsic and low codimensional there. Here, on the other hand, we rely mainly on the assumption that the sectional curvature is nonpositive.

Come back to our proof of Theorem 3 , for $T \in \mathcal{F}$, extend $C_{T}$ linearly over $\mathbf{C}$ to the complexification

$$
\mathcal{F}^{\perp} \otimes \mathbf{C}=\mathcal{L}^{\perp} \oplus \overline{\mathcal{L}^{\perp}}
$$


Choose a local frame $\left\{e_{i}, \overline{e_{i}}\right\}_{i=1}^{r}$ such that each $e_{i} \in \mathcal{L}^{\perp}$. Write $C_{T}\left(e_{i}\right)=A_{i j} e_{j}+$ $\overline{B_{\bar{i} j}} \overline{e_{j}}$, then the matrix of $C_{T}$ under the basis $\{e, \bar{e}\}$ is

$$
C_{T}=\left[\begin{array}{ll}
A & \bar{B} \\
B & \bar{A}
\end{array}\right], \quad \text { so } \quad C_{J T}=J C_{T}=\sqrt{-1}\left[\begin{array}{ll}
A-\bar{B} \\
B-\bar{A}
\end{array}\right]
$$

where $J$ is the almost complex structure of $M$. For any real numbers $a$ and $b$, write $\lambda=a+\sqrt{-1} b$, we have

$$
C_{a T+b J T}=\left[\begin{array}{ll}
\lambda A & \overline{\lambda B} \\
\lambda B & \overline{\lambda A}
\end{array}\right] .
$$

So we know that the above matrix has no non-zero real eigenvalue for any $\lambda \in \mathbf{C}$.

In the case when the dimension of $\mathcal{L}^{\perp}$ is 1 , that is, when $A$ and $B$ are $(1 \times 1)$ metrices, it is easy to see that this condition implies that $A=B=0$.

Our goal is to show that $A=B=0$ for any $T \in \mathcal{F}$ in general. This means that the leaves of $\mathcal{L}$ are all parallel to each other, thus $M=\mathbf{C}^{n-r} \times N^{r}$ by the real analyticity of the metric. We could conclude that $A=B=0$ if we can find a basis $\left\{e_{i}\right\}$ for $\mathcal{L}^{\perp}$ under which both $A$ and $B$ are diagonal matrices.

Let us fix a generic point $p \in U$, and take a local tangent frame $\left\{e_{i}, e_{\alpha}\right\}$ of type $(1,0)$ near $p$, such that each $e_{i} \in \mathcal{L}^{\perp}$ and each $e_{\alpha} \in \mathcal{L}$. We will fix the range of indices as follows.

$$
1 \leq i, j, \ldots \leq r ; \quad r+1 \leq \alpha, \beta, \ldots \leq n .
$$

Denote by $\theta, \Theta$ the matrices of the connection and the curvature under the frame $e$. We have

$$
d \varphi=\varphi \wedge \theta, \quad d \theta=\Theta+\theta \wedge \theta
$$

where $\varphi=\left(\varphi^{1}, \ldots, \varphi^{n}\right)$ is the row vector of the coframe dual to $e$. We have

$$
\theta_{\alpha}^{i}=-\sum_{k}\left(A_{k}^{i} \varphi^{k}+B_{\bar{k}}^{i} \overline{\varphi^{k}}\right)
$$

where $A, B$ appear in the matrix of $C_{T}$ for $T=e_{\alpha}+\overline{e_{\alpha}}$ as before. Here and below $\alpha$ is fixed. Note that if we change the basis $\left\{e_{i}\right\}$ to a new one $\left\{\epsilon_{i}\right\}$ with $\epsilon_{i}=\sum_{j} P_{i}^{j} e_{j}$, then the matrices $A$ and $B$ are replaced by $P A P^{-1}$ and $P B P^{-1}$ under the new basis $\left\{\epsilon_{i}\right\}$ of $\mathcal{L}^{\perp}$.

Write $\Omega_{a \bar{b}}=\sum_{c=1}^{n} \Theta_{a}^{c} g\left(e_{c}, e_{b}\right)$. Then

$$
\Omega_{a \bar{b}}=R_{a \bar{b} c \bar{d}} \varphi^{c} \wedge \overline{\varphi^{d}}
$$

where $R$ are the components of the curvature under the frame $e$. By our definition of $\mathcal{L}$ and the nonpositivity of the sectional curvature, we know that $R_{a \bar{b} c \bar{d}}=0$ unless all four indices are in the range between 1 and $r$ (cf. [Z]). The second Bianchi identity then implies that

$$
\sum_{j=1}^{r} \theta_{\alpha}^{j} \wedge \Omega_{j \bar{k}}=0
$$


for any $\alpha$ and $k$. That is,

$$
\sum_{i, j} A_{\alpha i}^{j} \varphi^{i} \wedge \Omega_{j \bar{k}}=0, \quad \sum_{i, j} B_{\alpha \bar{i}}^{j} \overline{\varphi^{i}} \wedge \Omega_{j \bar{k}}=0
$$

or equivalently,

$$
\begin{aligned}
& \sum_{j}\left(A_{i}^{j} R_{j \bar{k} p \bar{l}}-A_{p}^{j} R_{j \bar{k} i \bar{l}}\right)=0 \\
& \sum_{j}\left(B_{\bar{i}}^{j} R_{j \bar{k} p \bar{l}}-B_{\bar{l}}^{j} R_{j \bar{k} p \bar{i}}\right)=0
\end{aligned}
$$

for any $i, p, k, l$. Here we dropped the reference in $\alpha$, which is understood to be fixed in the discussion below.

First we claim that $A$ is diagonalizable. Assume not, then by the Jordan decomposition, there will be non-zero row vectors $x$ and $y$ such that

$$
x A=\lambda x+y, \quad y A=\lambda y
$$

for some constant $\lambda$. Multiply (1) by $x_{p} y_{i}$ and sum over $p$ and $i$, we get

$$
R_{(\lambda y) \bar{k} x \bar{l}}=R_{(\lambda x+y) \bar{k} y \bar{l}}
$$

for any $k, l$. That is, $R_{y \bar{k} y \bar{l}}=0$ for any $k, l$. In particular, we have $R_{y \bar{y} y \bar{y}}=0$. Since the sectional curvature is nonpositive, this implies that $R_{y \bar{y} z \bar{z}}=0$ for any other tangent vector $z$ (cf. [Z]), thus the Ricci curvature in the direction $y$ would be zero, a contradiction. Therefore the matrix $A$ must be diagonalizable.

Write $\mathcal{L}_{p}^{\perp}=V_{1} \oplus \cdots \oplus V_{s}$ for the decomposition into eigenspaces of $A$, that is, on each $V_{a}$, the linear transformation $A$ is a constant $\lambda_{a}$ times the identity, and $\lambda_{a} \neq \lambda_{b}$ for any $1 \leq a<b \leq s$. For any $a \neq b$ and any $x \in V_{a}, y \in V_{b},(1)$ gives

$$
R_{x \bar{*} \bar{*}}=0 \text {. }
$$

Multiply (2) by $\overline{x_{i} y_{k}}$ and sum over $i, k$, and using the above identity, we have

$$
\sum_{j} B_{\bar{x} j} R_{j \bar{y} p \bar{l}}=0
$$

for any $p, l$. Contracting over $p$ and $l$, we get

$$
\sum_{j} B_{\bar{x} j} r_{j \bar{y}}=0
$$

where $r=\left(r_{i \bar{j}}\right)$ stands for the Ricci tensor. Since the Ricci is negative definite on $\mathcal{L}^{\perp}$, this means that $B_{\bar{x} y}=0$. That is, $B$ respects the decomposition $\mathcal{L}_{p}^{\perp}=$ $V_{1} \oplus \cdots \oplus V_{s}$, and so does the Ricci tensor.

Fix a block $V_{a}$, in it the equation (2) gives $B r={ }^{t}(B r)$. Since $r$ is negative definite, we can choose a basis $e^{(a)}$ of $V_{a}$ under which $r=-I$, so $B$ becomes a complex symmetric matrix. Since for any complex symmetric matrix $B$, there exists a unitary matrix $Q$ such that ${ }^{t} Q B Q$ is diagonal, note that this is just $\bar{P} B P^{-1}$ 
for $P=Q^{*}$, so we have a new basis of $V_{a}$ under which $B$ is diagonal. In the meantime, $A=\lambda_{a} I$ becomes $P A P^{-1}=\lambda_{a} I$ and remains to be diagonal. So there exists a basis of $\mathcal{L}_{p}^{\perp}$ under which $A$ and $B$ are both diagonal matrices. Now the condition that $C_{a T+b J T}$ can not have any non-zero real eigenvalue for any real numbers $a$ and $b$ implies that $A=B=0$. This completes the proof of Theorem 3 .

\section{Discussions and examples}

In this section, let us discuss some issues relating to Theorem 1 and Theorem 3 . Let us start with the real analyticity assumption.

Our proof of Theorem 1 and Theorem 3 rely on the extra assumption that the metric $g$ is real analytic. If we drop that assumption in Theorem 3, then the proofs show that any connected component $U_{a}$ of $U \subset M$ must be isometric to a product $\mathbf{C}^{n-r} \times N_{a}$, where $N_{a}$ is a piece of Kähler manifold of dimension $r$ that has nonpositive sectional curvature and negative Ricci curvature.

We do not know if there is any example of Cartan-Hadamard Kähler manifold $M^{n}$ with Ricci rank $r<n$, but without global Euclidean de Rham factor. Our proof here only shows that such a manifold will have local Euclidean de Rham factors. In other words, if one drops the real analyticity condition in Theorem 3, we do not know if the conclusion will still be true. It is conceivable that one might be able to 'patch up' things to get a manifold without global flat factor.

If one requires that this $M$ is isometrically the universal covering space of a compact manifold, however, we believe the leaves of $\mathcal{L}$ must close up in the quotient, so the real analyticity assumption should be just a technical one in Theorem 1, and the theorem should hold true without this extra assumption. The key here is have a generalization of Eberlein's theorem to local Euclidean de Rham factors, but at this point we do not know how to deal with it.

In the low dimensional cases, Theorem 1 does hold true without the real analyticity assumption. For example, if $\left(M^{2}, g\right)$ is a compact Kähler surface with nonpositive sectional curvature, then by the classification theory for surfaces and the fact that $M$ can not contain any rational curve, we know that a finite cover of $M^{2}$ must be either a complex 2-torus, a product of the complex 1-torus with a curve of genus $\geq 2$, or a general type surface. The Kodaira dimension is always equal to the Ricci rank or the codimension of the Euclidean de Rham factor.

Similarly, in dimension 3, Theorem 1 also holds without the real analyticity assumption.

Next, let us consider the completeness assumption in Theorem 3. We would like to point out that, without the completeness assumption, Theorem 3 is not true, even in dimension 2. That is, if $(U, g)$ is a piece of Kähler surface with real analytic metric $g$, nonpositive sectional curvature, and with Ricci tensor of rank 1 everywhere on $U$, then the leaves of the kernel foliation of the Ricci are not 
necessarily parallel to each other. (In fact, this foliation in general may not even be holomorphic.)

This phenomenon was discussed in details in the paragraph (7.1) on p. 595 of the paper by Mok $([\mathrm{M}])$. The referee suggested the following simple but illustrative example.

Example 1. Let $f: \mathbf{C} \rightarrow \mathbf{C}^{3}$ be a holomorphic map such that $f^{\prime} \wedge f^{\prime \prime} \wedge f^{\prime \prime \prime} \neq 0$. For instance, one could let $f$ be $f(z)=\left(z, z^{2}, z^{3}\right)$. Consider the holomorphic map $F: U=\mathbf{C} \times \mathbf{C}^{*} \rightarrow \mathbf{C}^{3}$ defined by $F(z, t)=f(z)+t f^{\prime}(z)$. The assumption on $f$ implies that $F$ is an immersion. Denote by $g$ the $F$-pull back of the Euclidean metric on $\mathbf{C}^{3}$, then $\left(U^{2}, g\right)$ is a (non-complete) Kähler surface, with nonpositive bisectional curvature.

Denote by $B$ the second fundamental form of $U$ in $\mathbf{C}^{3}$, and write

$$
T=F_{*}\left(\frac{\partial}{\partial t}\right)=f^{\prime}, \quad Z=F_{*}\left(\frac{\partial}{\partial z}\right)=f^{\prime}+t f^{\prime \prime} .
$$

The tangent space of $U$ is spanned by $\{T, Z\}$, or equivalently $\left\{f^{\prime}, f^{\prime \prime}\right\}$. We have

$$
B(T, T)=B(T, Z)=0, \quad B(Z, Z)=t\left(f^{\prime \prime \prime}\right)^{\perp} \neq 0 .
$$

So under the frame $\{T, Z\}$, the only non-zero component of the curvature tensor is $R_{Z \bar{Z} Z \bar{Z}}=-|B(Z, Z)|^{2}<0$. Thus $\left(U^{2}, g\right)$ has nonpositive sectional curvature as well.

The kernel foliation of the Ricci is just the developable (meaning the tangent space of $U$ is constant along each ruling) ruling foliation spanned by $T$, its leaves are not all parallel to each other since $f^{\prime} \wedge f^{\prime \prime} \neq 0$.

This example illustrates the necessity of the completeness assumption in Theorem 3 .

Finally, let us consider the Riemannian counterpart of Theorem 3. Note that the strict Riemannian analogue of Theorem 3 does not hold, namely, there exists (real analytic) Cartan-Hadamard manifold $M^{n}$ without Euclidean de Rham factor such that the Ricci rank is less than $n$. To see such an example, consider the the following cubic threefold

Example 2. Let $M^{3} \subseteq \mathbf{R}^{4}$ be the graph of the function

$$
w=f(x, y, t)=\left(x^{2}-y^{2}+2 t x y\right) /\left(1+t^{2}\right)
$$

over $\mathbf{R}^{3}$. It is easy to see that $M^{3}$ is ruled along the $t$ direction. In fact, it is developable (i.e., the tangent space of $M^{3}$ along each ruling line is constant). The Ricci rank is equal to 2. The second fundamental form of $M^{3}$, which is given by the Hessian of $f$, has one positive, one negative and one zero eigenvalue everywhere. Therefore, $M$ has nonpositive sectional curvature. Clearly, these rulings are not all parallel to each others.

Similarly, we may consider the following developable Euclidean submanifold 
Example 3. Let $M^{2 k+1} \subset \mathbf{R}^{3 k+1}$ be the graph of the smooth map $F: \mathbf{R}^{2 k+1} \rightarrow$ $\mathbf{R}^{k}$ defined by

$$
F(t, x, y)=2 t x-t^{2} y, \quad(t, x, y) \in \mathbf{R} \times \mathbf{R}^{k} \times \mathbf{R}^{k} .
$$

Equip $M$ with the restriction of the standard Euclidean metric. Then $M^{n}$ is a real analytic Cartan-Hadamard manifold without Euclidean de Rham factor, and has Ricci rank $r=k+1$. So $r$ satisfies the equality $n=2 r-1$. In fact, $M^{n}$ even has nonpositive curvature operator. We will omit the details of the verification here, since it's straight forward computation.

The above examples show that, on a given Cartan-Hadamard manifold $M^{n}$ (i.e., a complete, simply-connected Riemannian manifold with nonpositive sectional curvature), the Ricci rank $r$ can be less than $n$ even when $M^{n}$ is real analytic and is without Euclidean de Rham factor. However, this $r$ cannot be too small relative to $n-s$, where $s$ is the so-called core number, which measures the 'flatness' of the manifold. In fact, $r$ has to be bigger than half of $n-s$, and this inequality is sharp by Example 3, where $s=0$. We refer the readers to [G-Z] for the details. Compare this to the special but related case when $M^{n}$ is a submanifold in $\mathbf{R}^{n+p}$ for small $p$, where similar questions were studied [F], [F-Z], [F-Z1].

\section{Proof of Theorem 2}

In this section, we shall prove Theorem 2 stated in the introduction. First let us start with some discussion about the conjecture itself.

Let $M^{n}$ be a Kähler manifold with nonpositive sectional curvature. First of all, notice that if a tangent direction $v$ has zero holomorphic sectional curvature, then it will have zero Ricci curvature as well. For a proof of this see [Z]. Secondly, it is easy to see that $M$ does not contain any rational curve, smooth or singular. In fact, when $M$ is complete and $N$ is any compact complex manifold with $\pi_{1}=0$ (or finite), then any holomorphic map from $N$ into $M$ must be trivial because the universal cover of $M$ is Stein, by a theorem of $\mathrm{Wu}$ [W]. Also, if $E$ is an elliptic curve in $M$, the curvature decreasing property for holomorphic subbundles will force $E$ to be smooth, flat, and totally geodesic in $M$. So the Ricci curvature in the tangent direction of $E$ is zero. Thus $E \cdot K_{M}=0$, where $K_{M}$ is the canonical line bundle of $M$.

As a consequence, if $M^{n}$ is a general type, compact Kähler manifold with nonpositive sectional curvature, then it does not contain any elliptic curve (since otherwise $K_{M}$ will not be ample, thus there will be a rational curve in $M$ by a result of Kawamata $[\mathrm{K}])$.

By a theorem of Brody, a compact complex manifold $M$ will be Kobayashi hyperbolic if any holomorphic map from $\mathbf{C}$ into $M$ is constant. So Conjecture 2 is in a way a complex analogue of the Bangert-Schroeder theorem [B-S], which states that for a compact, real analytic, nonpositively curved Riemannian manifold $M$, if 
the universal covering space of $M$ contains a flat (of maximal possible dimension in $M$ ), then $M$ will contain a closed flat (i.e., a totally geodesic flat torus) of the same dimension.

Theorem 2. Let $M^{2}$ be a compact Kähler surface of nonpositive sectional curvature. If it is a general type surface, then it will be Kobayashi hyperbolic.

Proof. First of all, such a surface has ample canonical line bundle $K_{M}$ and does not contain any (smooth or singular) rational or elliptic curves. (In the elliptic case, the curvature condition forces the curve to be smooth, and has zero intersection number with $K_{M}$.)

By the result of [Z], we know that the two Chern numbers of $M$ satisfy $c_{1}^{2} \geq 2 c_{2}$. When $c_{1}^{2}>2 c_{2}$, one can apply the result of $\mathrm{Lu}$ and Yau [L-Y] to conclude that any entire holomorphic map $f: \mathbf{C} \rightarrow M$ degenerates, i.e., its image must be contained in a curve, necessarily a rational or elliptic one. That proves the hyperbolicity of $M$.

When $c_{1}^{2}=2 c_{2}$, one can still apply the result of $[\mathrm{L}-\mathrm{Y}]$, except the case when there is a horizontal surface $Y$ contained in $\mathbf{P}\left(T_{M}\right)$, which satisfies $Y \cdot L^{2}=0$. Here $L$ is the dual of the tautological line bundle (see paragraph 3.2 of $[\mathrm{L}-\mathrm{Y}]$ ). In the latter case, the cotangent bundle $\Omega_{M}$ of $M$ is not stable under the polarization $K_{M}$. On the other hand, by [Y] $M$ admits a Kähler-Einstein metric with negative Ricci curvature. So $\Omega_{M}$ is the direct sum of two line bundles, and the Kähler-Einstein metric splits. Hence the universal cover $\widetilde{M}$ is a product, which is necessarily the bidisc. In this case the hyperbolicity is well known.

Acknowledgment. We would like to thank the referee of this article for the very detailed report, which contains Example 1 and many other suggestions, that improved the presentation and made it more readable, especially in the proof of Theorem 3. We are very grateful to the help.

\section{References}

[A] K. Abe, Applications of a Riccati type differential equation to Riemannian manifolds with totally geodesic distributions, Tôhoku Math J. 25 (1973), 425-444.

[A-S] M. Anderson and V. Schroeder, Existence of flats in manifolds of nonpositive curvature, Invent. Math. 85 (1986), 303-315.

[B] W. Ballmann, Nonpositively curved manifolds of higher rank, Ann. Math. 122 (1985), 597-609.

[B-G-S] W. Ballmann, M. Gromov and V. Schroeder, Manifold of nonpositive curvature, Progress in Mathematics, vol. 61, Birkhäuser, Basel, 1985.

[B-S] V. Bangert and V. Schroeder, Existence of flat tori in analytic manifolds of nonpositive curvature, Ann. Sci. École Normale Sup. 24 (1991), 605-634.

[Br] R. Brody, Compact manifolds and hyperbolicity, Trans. Amer. Math. Soc. 235 (1978), 213-219. 
[Bu-S] K. Burns and R. Spatzier, Manifolds of nonpositive curvature and their buildings, Publ. Math. IHES 65 (1987), 35-59.

[D-R] M. Dajczer and L. Rodriguez, Complete real Kähler minimal submanifolds, J. reine angew. Math. 419 (1991), 1-8.

[E1] P. Eberlein, A canonical form for compact nonpositively curved manifolds whose fundamental groups have nontrivial center, Math. Ann. 260 (1982), 23-29.

[E2] P. Eberlein, Euclidean de Rham factor of a lattice of nonpositive curvature, J. Diff. Geom. 18 (1983), 209-220.

[E3] P. Eberlein, Isometry groups of simply connected manifolds of nonpositive curvature, II, Acta Math. 149 (1982), 41-69.

[E4] P. Eberlein, Symmetry diffeomorphism groups of a manifold of nonpositive curvature, II, Indiana Math. J. 37 (1988), 735-752.

[E-H-S] P. Eberlein, U. Hamenstädt and V. Schroeder, Manifolds of nonpositive curvature, Differential geometry: Riemannian geometry (Los Angeles, CA, 1990), 179-227, Proc. Sympos. Pure Math., 54, Part 3, Amer. Math. Soc., Providence, RI, 1993.

[Fe] D. Ferus, On the completeness of nullity foliations, Michigan Math. J. 18 (1971), 61-64.

[F] L. Florit, On submanifolds with nonpositive extrinsic curvature, Math. Ann. 298 (1994), $187-192$.

[F-Z] L. Florit and F. Zheng, On nonpositively curved Euclidean submanifolds: splitting results, Comm. Math. Helv. 74 (1999), 53-62.

[F-Z1] L. Florit and F. Zheng, On nonpositively curved Euclidean submanifolds: splitting results, II, J. Reine Angew. Math. 508 (1999), 1-15.

[Fr] S. Frankel, Locally symmetric and rigid factors for complex manifolds via harmonic maps, Ann. of Math. 141 (1995), 285-300.

[G] M. Gromov, Kähler hyperbolicity and $L^{2}$-Hodge theory, J. Diff. Geom. 33 (1991), 263292 .

[G1] M. Gromov, Hyperbolic groups, Essays in group theory (S. M. Gersten, ed.), MSRI Publ. 8, Springer-Verlag, Berlin, Heidelberg, New York, 1987, 75-264.

[G-Z] D. Guler and F. Zheng, On Ricci rank of Cartan-Hadamard manifolds, to appear in International J. Math.

[H-S] C. Hummel and V. Schroeder, Tits geometry associated with 4-dimensional closed realanalytic manifolds of nonpositive curvature, J. Diff. Geom. 48 (1998), 531-555.

[L-Y] S. Lu and S. T. Yau, Holomorphic curves in surfaces of general type, Proc. Natl. Acad. Sci. USA 87 (1990), 80-82.

[K] Y. Kawamata, On the length of an extremal rational curve, Invent. Math. 105 (1991), 609-611.

[M] N.-M. Mok, Factorization of semisimple discrete representations of Kähler groups, Invent. Math. 110 (1992), 557-614.

[M1] N.-M. Mok, Metric rigidity theorems on Hermitian locally symmetric manifolds, Series in Pure Mathematics, 6. World Scientific, Inc., Teaneck, NJ, 1989.

[M2] N.-M. Mok, Topics in complex differential geometry, Recent topics in differential and analytic geometry, 1-141, Adv. Stud. Pure Math., 18-I, Academic Press, Boston, MA, 1990.

[MSY] N.-M. Mok, Y.-T. Siu and S.-K. Yeung, Geometric superrigidity, Invent. Math. 113 (1993), 57-83.

[S] V. Schroeder, Existence of immersed tori in manifolds of nonpositive curvature, J. Reine Angew. Math. 390 (1988), 32-46.

[S1] V. Schroeder, Structure of flat subspaces in low-dimensional manifolds of nonpositive curvature, Manuscripta Math. 64 (1989), 77-105.

[S2] V. Schroeder, Codimension one tori in manifolds of nonpositive curvature, Geom. Dedicata 33 (1990), 251-263.

[S3] V. Schroeder, Analytic manifolds of nonpositive curvature with higher rank subspaces, Arch. Math. (Basel) 56 (1991), 81-85. 
[S-S] B. Shiffman and A. Sommese, Vanishing theorems on complex manifolds, Progress in Math., vol. 56, Birkhäuser, Boston, 1985.

[Si] Y.-T. Siu, The complex analyticity of harmonic maps and the strong rigidity of compact Kähler manifolds, Ann. Math. 112 (1980), 73-111.

[W] H. Wu, Negatively curved Kähler manifolds, Notices Amer. Math. Soc. 14 (1967), 515.

[Y] S. T. Yau, Calabi's conjecture and some new results in algebraic geometry, Proc. Nat. Acad. Sci. USA 74 (1977), 1798-1799.

[Z] F. Zheng, First Pontrjagin form, rigidity and strong rigidity of nonpositively curved Kähler surfaces of general type, Math. Zeit. 220 (1995), 159-169.

[Z1] F. Zheng, Examples of nonpositively curved Kähler manifolds, Comm. Anal. Geom. 4 (1996), 129-160.

[Z2] F. Zheng, Hirzebruch-Kato surfaces, Deligne-Mostow's construction, and new examples of negatively curved compact Kähler surfaces, Comm. Anal. Geom. 7 (1999), 755-786.

Fangyang Zheng

The Ohio State University

Department of Mathematics

231 West 18th Avenue

Columbus, OH 43210-1174

USA

e-mail: zheng@math.ohio-state.edu

(Received: February 29, 2000)

(1) To access this journal online:

(20) http://www.birkhauser.ch 